\title{
Pre-selection of primary intubation technique is associated with a low incidence of difficult intubation in patients with a BMI of $35 \mathrm{~kg} / \mathrm{m}^{2}$ or higher
}

\author{
Tiberiu Ezri $^{1 *}$, Ronen Waintrob ${ }^{2}$, Yuri Avelansky ${ }^{1}$, Alexander Izakson ${ }^{3}$, Katia Dayan ${ }^{4}$, Mordechai Shimonov $^{5}$ \\ ${ }^{1}$ Department of Anesthesia, ${ }^{4}$ Surgery B and ${ }^{5}$ Surgery A, Wolfson Medical Center, Holon, affiliated to Sackler Medical School, Tel Aviv \\ University, Israel \\ ${ }^{2}$ Department of Anesthesia, Poriya Medical Center, Tiberias and ${ }^{3}$ Ziv Medical Center, Safed, Israel, Azrieli Faculty of Medicine, Bar-Ilan \\ University, Safed, Israel \\ * Outcomes Research Consortium, Cleveland, OH, USA
}

\begin{abstract}
Background: The incidence of difficult intubation (DI) in obese patients may reach a two-digit figure. No studies have assessed the effect of primary use of special intubation devices on lowering the incidence of DI. We assessed the effect of primary selection of special intubation techniques on the incidence of DI in patients with a BMI of $35 \mathrm{~kg} / \mathrm{m}^{2}$ or higher.

Patients and methods: Data from 546 patients with a BMI of $35 \mathrm{~kg} / \mathrm{m}^{2}$ or higher who underwent bariatric surgery at Wolfson Medical Center from 2010 through 2014 was retrospectively extracted and analyzed for demographics, predictors of DI and intubation techniques employed. Difficult intubation was defined as the presence of at least one of the followings: laryngoscopy grade 3 or 4 , need for $>1$ laryngoscopy or intubation attempt, need for changing the blade size, failed direct laryngoscopy (DL), difficult or failed videolaryngoscopy (VL-Glidescope), difficult or failed awake fiberoptic intubation (AFOI) and using VL or awake AFOI as rescue airway techniques. Primary intubation techniques were direct DL, VL and AFOI. We correlated the predictors of DI with the actual incidence of DI and with the choice of intubation technique employed.

Results: The overall incidence of DI was $1.6 \%(1.5 \%$ with DL vs. 2.2 with VL + AFOI, $p=0.61)$. With logistic regression analysis, age was the only significant predictor of DI. Predictors of DI that affected the selection of VL or AFOI as primary intubation tools were Mallampati class 3 or 4, limited neck movement, age, male gender, body mass index and obstructive sleep apnea syndrome.

Conclusion: The lower incidence of DI in our study group may stem from the primary use of special intubation devices, based on the presence of predictors of DI.

Keywords: difficult airway, obese patients, intubation technique

\section{Introduction}

The definition of difficult intubation (DI) is debatable. This may affect the reported incidence of DI. Recently, Ramachandran and Klock [1] defined DI as

Alexander Izakson, MD Ziv Medical Center Azrieli Faculty of Medicine Bar-Ilan University Henrietta Szold st. 8, POB 1589 Safed, Israel

E-mail: Alexander.i@ziv.health.gov.il

one or more of the following: difficult direct laryngoscopy, difficult video laryngoscopy, or difficult flexible bronchoscopic visualization. Brodsky et al. [2] defined an intubation as problematic if the product of the Cormack-Lehane laryngoscopy view grade and the number of intubation attempts was $\geq 3$. Though with several flaws (i.e. the subjective assessment of lifting force during laryngoscopy, the fact that the Sellick maneuver - cricoid pressure is not scored as external laryngeal pressure and using twice the "invisible cords" in two different scoring parameters), the intubation difficulty scale (IDS) [3] which is composed of seven score parameters is the most comprehensive and most frequently method employed to define DI.
\end{abstract}


As a result of a lack of a standardized DI definition, a wide range of DI incidences have been reported. This is even more evident in obese and morbidly patients which may have more predictors of DI as compared to lean patients [3]. It is well known that obese and morbidly obese patients have a higher incidence of DI. However, it is very concerning that the incidence of DI in these patients may reach two digit figures [3]. Such a high incidence of DI may lead to life-threatening situations and may call for preventative measures to significantly lower the incidence of DI in obese patients. DI may be associated with lifethreatening complications such as aspiration of gastric contents and severe hypoxemia. Nevertheless, even a recent study [4] recommends the preparation of rescue airway devices with a high suspicion of DI instead of the pre-selection of primary intubation techniques if such a scenario is predicted.

In this study, we hypothesized that the pre-selection of special primary intubation techniques based on preexisting DI predictors may significantly decrease the incidence of DI in patients with a BMI of $35 \mathrm{~kg} / \mathrm{m}^{2}$ or higher when compared to the figures published in the literature.

\section{Methods}

This retrospective study was approved by our hospital's IRB (Helsinki) committee. After excluding 49 patients (only in 40 patients was there documentation of using intubation in the "ramp" position and another 9 patients with incomplete information in their records) that could have been an important source of bias and to reduce the confounding effects, data from the paper records of 546 patients with a BMI of $35 \mathrm{~kg} / \mathrm{m}^{2}$ or higher who underwent bariatric surgery at Wolfson Medical Center during a five-year period, from 2010 through 2014 was extracted and analyzed. There were no missing records. Patients with a history of difficult intubation were excluded from the data analysis. Analyzed data included demographics (age and gender), co-existing obstructive sleep apnea syndrome (OSAS), body mass index (BMI), predictors of DI and intubation techniques employed. Predictors of DI were considered limited neck mobility, limited mouth opening $(<3 \mathrm{~cm})$, short thyromental distance $<6 \mathrm{~cm}$, Mallampati class 3 (soft palate, base of uvula visible.) or 4 (only hard palate visible) and dentition problems (loose or prominent upper teeth). Obstructive sleep apnea syndrome (OSAS) was also considered risk factors for DI.

Difficult intubation was defined as the presence of at least one of the following: laryngoscopy view grade (Cormack-Lehane) 3 (only epiglottis seen, none of glottis seen) or 4 (neither glottis nor epiglottis seen), need for $>1$ laryngoscopy or intubation attempt, need for changing the blade size, difficult or failed videolaryngoscopy (VL), difficult or failed awake fiberoptic intubation (AFOI) and using VL or awake AFOI as rescue airway techniques.

The choices of primary intubation techniques were direct laryngoscopy (DL- curved blade Macintosh \#3), VL (Glidescope, Verathon Inc., 20001 North Creek Pkwy, Bothell, WA 98011, US) and AFOI (Olympus Corporation of the Americas, 3500 Corporate Parkway, Center Valley, PA 18034, US).

Preoxygenation was followed by anesthesia induced with propofol, with or without rapid sequence induction (RSI), based upon the attending anesthesiologist's decision. Muscle relaxation for endotracheal intubation was provided with either succinylcholine $(1 \mathrm{mg} / \mathrm{kg}$ total body weight [5]) for both RSI and non-RSI or with rocuronium $0.6 \mathrm{mg} / \mathrm{kg}$ for non-RSI and $1.2 \mathrm{mg} / \mathrm{kg}$ for RSI, dosed per ideal body weight [5].

As per our department's routine, AFOI or VL was used with suspicion of DI. The rest of the patients were intubated with DL. Special airway devices such as the Glidescope and fiberoptic bronchoscope were readily available in all cases. Rescue VL and AFOI were not counted as primary intubation techniques.

Data were compared between two groups of patients. The first group comprised patients who were primarily intubated with DL and the second group those who were primarily intubated with either VL or AFOI.

The primary outcome was the overall incidence of DI and the incidence of DI with DL vs. VL + AFOI. Secondary outcomes were the correlation between the DI predictors and the chosen intubation technique (DL or AFOI $+\mathrm{VL}$ ) and the correlation between the DI predictors and the incidence of DI.

\section{Data Analysis}

Sample size justification: A sample size of 433 would provide $80 \%$ power to detect a true difference from a known proportion; specifically, we sought to compare our difficult airway prevalence $(1.6 \%)$ against the lowest value we found in the literature $(3.5 \%$ - see Ref. 4 using the comparison of two proportions - a given proportion against a known value). This considers a two-sided alpha of 0.05 . In actuality, we reviewed 546 cases included in this report, indicating an adequate sample size to address the primary hypothesis.

Data were stored on Excel spreadsheet (Microsoft, USA) and analyzed on SPSS Statistical Analysis Software (IBM, USA). Distributions of continuous variables were tested for normality using the Kolmogorov-Smirnov test and described as mean \pm standard deviation. Nominal variables were summarized in frequency tables and presented as $\mathrm{n}(\%)$. Continuous 
variables were compared by intubation technique or difficult intubation using the t-test for independent samples. Categorical variables were compared by intubation technique or difficult intubation using the chi square test. Logistic regression analysis was used to model difficult intubation (with age, sex and BMI as predictors) and odds ratios with $95 \%$ confidence intervals were calculated. All tests were two sided and considered significant at $\mathrm{p}<0.05$.

\section{Results}

Table 1 presents the demographic data and the prevalence of DI by the intubation technique. Eighty four percent of the patients had DL and $16 \%$ had special intubation techniques. Of these, $88.5 \%$ ( 77 cases $)$ were primarily intubated with VL and $11.5 \%$ (10 cases) with AFOI. The overall incidence of difficult intubation was $1.6 \%$. There were seven cases of DI in the DL group $(1.5 \%)$ and two cases $(2.2 \%)$ in the VL + AFOI group. In the DL group there were two failed intubations both owing to a grade $4 \mathrm{DL}$ view. Both patients' tracheas were successfully intubated with VL (rescue). There was one failed intubation + ventilation with VL used as primary intubation tool that ended in emergency tracheostomy. The patient's outcome was good. There was another failed primary VL with easy ventilation. The patient was awakened and intubated with AFOI (rescue). There was no failed AFOI.

Table 2 shows the correlations between DI predictors and risk factors and the actual occurrence of DI. Only age was a significant predictor of DI while male gender was a marginally significant predictor of DI ( $p$ $=0.057$ ). Logistic regression was used to model difficult intubation. The model was significant $(\mathrm{p}=0.029)$ and correctly classified $98.3 \%$ of the study population to difficult intubation. Age was the only significant predictor, OR $1.06(95 \%$ CI $1.003-1.120, \mathrm{p}=0.037)$ indicating that each additional year of age increases odds of difficult intubation by a relative $6 \%$.

Table 3 presents the correlations between DI predictors and risk factors and the choice of primary intu-

Table 1. Demographic data and prevalence of difficult intubation by intubation technique

\begin{tabular}{|lccc|}
\hline Intubation technique & $\begin{array}{c}\text { Direct } \\
\text { laryngoscopy }\end{array}$ & $\begin{array}{c}\text { Special intubation techniques } \\
\text { (videolaryngoscopy }+ \text { awake fiberoptic intubation) }\end{array}$ & $p$ \\
\hline Variable & $459(84.1)$ & $77+10=87(15.9)$ & 0.000 \\
Number of patients (\%) & $40 \pm 13$ & $47 \pm 12$ & 0.000 \\
\hline Age (years) & 27.5 & 52.9 & 0.000 \\
Males sex (\%) & $42 \pm 4.9$ & 457 & 0.001 \\
Body mass index $\left(\mathrm{kg} / \mathrm{m}^{2}\right)$ & 9.4 & 21.8 & 0.57 \\
Obstructive sleep apnea syndrome (\%) & 4.4 & 1.1 & 0.61 \\
\hline Gastro-esophageal reflux disease $(\%)$ & $7(1.5)$ & $2(2.2)$ & \\
\hline Difficult intubation $\mathrm{N}(\%)$ & & & \\
\hline
\end{tabular}

Results are presented as mean \pm standard deviation or percentage

Table 2. Correlations between DI predictors and risk factors and the actual occurrence of difficult intubation

\begin{tabular}{|lccc}
\hline & $\begin{array}{c}\text { Easy intubation } \\
\mathrm{N}=537\end{array}$ & $\begin{array}{c}\text { Difficult intubation } \\
\mathrm{N}=9\end{array}$ & $\mathrm{p}$ \\
\hline Difficult intubation predictors and risk factors & & & 0.031 \\
\hline Age (years) & $41 \pm 13$ & $51 \pm 11$ & 0,057 \\
Males sex (\%) & 31.1 & $41.6 \pm 4.5$ & 0.462 \\
Body mass index $\left(\mathrm{kg} / \mathrm{m}^{2}\right)$ & $42.9 \pm 5.4$ & 22.2 & 0.301 \\
Obstructive sleep apnea syndrome (\%) & 11.2 & 1.1 & 0.57 \\
Gastro-esophageal reflux disease (\%) & 4.4 & 44.4 & 0.222 \\
Mallampati 3 and $4(\%)$ & 26.3 & 0.0 & 0.629 \\
\hline Dentition problems $(\%)$ & 1.7 & 11.1 & 0.488 \\
Thyromental distance $<6 \mathrm{~cm}(\%)$ & 20.5 & 0.0 & 0.599 \\
\hline Mouth opening $<3 \mathrm{~cm}(\%)$ & 3.0 & 0.0 & 0.534 \\
\hline Limited neck movement $(\%)$ & 5.2 & & \\
\hline
\end{tabular}

Results are presented as mean \pm standard deviation or percentage

Logistic regression was used to model difficult intubation. The model was significant $(\mathrm{p}=0.029)$ and correctly classified $98.3 \%$ of the study population to difficult intubation. Age was the only significant predictor, OR $1.06(95 \%$ CI $1.003-1.120, p=0.037)$ indicating that each additional year of age increases the odds of difficult intubation by a relative $6 \%$. 
Table 3. Correlations between DI predictors and risk factors and choice of primary intubation technique

\begin{tabular}{|c|c|c|c|}
\hline Intubation technique & $\begin{array}{c}\text { Direct } \\
\text { laryngoscopy }\end{array}$ & $\begin{array}{c}\text { Special intubation techniques } \\
\text { (videolaryngoscopy }+ \text { awake } \\
\text { fiberoptic intubation) }\end{array}$ & $\mathrm{p}$ \\
\hline \multicolumn{4}{|c|}{ Difficult intubation predictors and risk factors } \\
\hline Age (years) & $40 \pm 13$ & $47 \pm 12$ & 0.000 \\
\hline Males sex $(\%)$ & 27.5 & 52.9 & 0.000 \\
\hline Body mass index $\left(\mathrm{kg} / \mathrm{m}^{2}\right)$ & 424.9 & 457 & 0.000 \\
\hline Obstructive sleep apnea syndrome (\%) & 9.4 & 21.8 & 0.001 \\
\hline Gastro-esophageal reflux disease (\%) & 4.4 & 1.1 & 0.57 \\
\hline Mallampati 3 and $4(\%)$ & 20.4 & 59.5 & 0.000 \\
\hline Dentition problems (\%) & 1.6 & 2.3 & 0.613 \\
\hline Thyromental distance $<6 \mathrm{~cm}(\%)$ & 20 & 21.8 & 0.703 \\
\hline Mouth opening $<3 \mathrm{~cm}(\%)$ & 2.4 & 5.7 & 0.089 \\
\hline Limited neck movement (\%) & 4.1 & 10.9 & 0.023 \\
\hline
\end{tabular}

Results are presented as mean \pm standard deviation or percentage

bation technique pre-selected by the anesthesiologist. The table shows that in choosing a special intubating device, anesthesiologists considered age, male sex, BMI, OSAS, Mallampati classes 3 and 4 and limited neck movement as risk factors for DI.

\section{Discussion}

Since clinicians may tend to primarily choose certain intubation techniques (i.e. AFOI), whenever a DI is suspected, it may be problematic to determine the true incidence of DI. Published randomized, controlled, prospective trials that estimated the incidence of DI did not allocate patients with suspected DI to special intubation techniques. This may increase the incidence of DI in obese patients and thus expose them to complications related to DI, such as aspiration of gastric content, severe hypoxemia, etc.

Our retrospective study provides an insight to the clinical practice of DI management in our 700 bed university hospital.

As previously stated, the incidence of DI in obese patients has not been clearly determined, though it is higher compared to lean patients and in many studies it reaches two digit figures ranging from $3.5 \%$ to $20.2 \%$ $[2,4,6-16]$. This wide range of reported DI in obese patients may reflect different definitions of DI. However, the high incidence of DI in obese patients may stem from risk factors specific to obesity (such as an abundance of soft tissues in the upper airway, OSAS), in addition to the presence of other DI predictors common to both lean and obese patients, such as Mallampati class 3 or 4, advanced age, male gender, limited neck extension and mouth opening, short thyromental distance and dentition problems.

Similarly to the uncertainty of the incidence of DI, the best way to predict DI in obese patients is also debatable.
Though high BMI has been traditionally considered as risk factor for DI in obese patients (16), many studies $[2,4,8,10,12,17]$ have questioned the reliability of BMI as a predictor of DI. Similarly, in our study population (see Table 2), the magnitude of BMI did not affect the incidence of DI.

This is consistent with the findings by Dohrn et al. [4] who demonstrated that BMI is not a risk factor for DI among obese patients. Curiously, in this study, the Cormarck-Lehane grade was considered a predictor of DL/DI instead of being considered as one of the definitions of DL/DI.

Other DI predictors as presented above were also unreliable in forecasting DI, except for a marginally significant effect of male gender $(p=0.057)$. Difficult intubation has been associated with increasing age in both obese and lean patients $[12,18]$. In our study, an increase in age was the single DI predictor associated with an increase in the incidence of DI $(\mathrm{p}=0.031)$. A logistic regression analysis used to model DI showed that the model was significant $(p=0.029)$ and correctly classified $98.3 \%$ of the study population to difficult intubation. Age was the only significant predictor, OR 1.06 (95\% CI 1.003-1.120, $\mathrm{p}=0.037$ ) indicating that each additional year of age increases the odds of difficult intubation by a relative $6 \%$.

Some obese patients may require special intubation techniques to mitigate the complications associated with DI. Brodsky et al. [2], considering that $6-7 \%$ of obese patients with a BMI $>40 \mathrm{~kg} / \mathrm{m}^{2}$ may require awake intubation. Two retrospective [7-8] studies have examined the elective use of AFOI in morbidly obese patients (in $4.2 \%$ and $2.7 \%$ of patients respectively). In their retrospective study, Hagberg et al. [7] have found that AFOI was used mainly in patients with a BMI $>60 \mathrm{~kg} / \mathrm{m}^{2}$ and in those with Mallampati classes 3 and 4. Two other studies [19-20], of small groups of obese patients have shown that VL improved a 
laryngoscopic grade view when compared to DL. However, none of these studies have estimated the incidence of DI rather the change in the laryngoscopic view.

The effect of pre-selection in obese patients of a specific intubation technique based on DI predictors on lowering the incidence of DI has not been investigated. Our hypothesis that pre-selection of special airway devices may decrease the incidence of DI in obese patients is reinforced by the data published by De Jong et al. [10]. In this study, VL was frequently used (in 38\% of DI cases) as a rescue intubation device rather than as a primary intubation tool. The use of specialized airway devices (such as AFOI or videolaryngoscopy) in obese patients was classified by the authors [9] as "definitive airway management techniques" that is, rescue tools after failed standard intubation. In a recent study of 1427 failed intubations, in a general surgical population (not specifically obese patients), Glidescope was the most successful (92\%) rescue intubation device [21]. These data may suggest that many of the patients who had DI and required a rescue change of the intubation technique could benefit from the primary use of VL or AFOI. This may also explain the lower incidence of DI in our patient population when compared to those in whom VL and FOI were used only as rescue tools [10] and not as primary airway devices. In a recent editorial [22] Asai calls for early use of VL with failed conventional laryngoascopy. Moreover, our retrospective study reveals that though the selection of primary intubating tool was at the discretion of the attending anesthesiologists, the choice for selection of VL or AFOI was based in most of patients on DI predictors commonly associated with DI (see Table 3 ) such as Mallampati 3 or 4 [2, 17, 23], older age [23] short thyromental distance [14] and male gender $[7,17,18]$. Although we found that OSAS was also considered a reason for using special intubation devices, Neligan et al. [17] found no correlation between OSAS and DI. The finding that special airway devices were more frequently used in our patients with higher BMIs suggests that our practitioners still consider BMI as a risk factor for DI as it is also the case in a few published studies [14, 16].

There are several potential limitations of our study. First, this was not a randomized, control trial. However, the study accurately reflects our department's clinical practice. A second limitation is that neck circumference was not measured and thus increased neck circumference was not considered a risk factor for DI. However, the predictive value of this measurement is still debated $[14,17]$. Another limitation is that intubation was not performed in an elevated head position ("ramp" position). However, though this position has shortened the time for laryngoscopy and intubation, it was not associated with fewer attempts at laryngoscopy and intubation when compared to the standard intubating position [24].

In conclusion, our data revealed that pre-selection of special devices such as videolaryngoscopy and awake fiberoptic intubation, as primary intubating tools in patients with predicted difficult intubation, was associated with a lower than the reported incidence of difficult intubation in patients with a BMI of $35 \mathrm{~kg} / \mathrm{m}^{2}$ or higher.

\section{Conflict of interest}

Nothing to declare

\section{References}

1. Ramachandran SK, Allan Klock P. Definition and incidence of the difficult airway. In: Hagberg CA (ed). Benumof and Hagberg's Airway Management. $3^{\text {rd }}$ ed. Philadelphia: Elsevier Saunders; 2013: 201-208

2. Brodsky JB, Lemmens HJ, Brock-Utne JG, Vierra M, Saidman LJ. Morbid obesity and tracheal intubation. Anesth Analg 2002; 94: 732-736. doi: 10.1097/00000539-200203000-00047

3. Adnet F, Borron SW, Racine SX, Clemessy JL, Fournier JL, Plaisance $P$, et al. The intubation difficulty scale (IDS): proposal and evaluation of a new score characterizing the complexity of endotracheal intubation. Anesthesiology 1997; 87: 1290-1297

4. Dohrn N, Sommer T, Bisgaard J, Rønholm E, Larsen JF. Difficult tracheal intubation in obese gastric bypass patients. Obes Surg 2016; 26: 2640-2647. doi: 10.1007/s1 1695-016-2141-0

5. De Baerdemaeker LEC, Van Limmen JGM, Van Nieuwenhove Y. How should obesity be measured and how should anesthetic drug dosage be calculated?. In: Leykin Y, Brodsky JB (eds.). Controversies in the anesthetic management of the obese surgical patient. 2013. Milan: Springer Verlag; 2013: 15-30. doi: 10.1007/ 978-88-470-2634-6 2

6. Lundstrřm LH, Møller AM, Rosenstock C, Astrup G, Wetterslev J. High body mass index is a weak predictor for difficult and failed tracheal intubation: a cohort study of 91,332 consecutive patients scheduled for direct laryngoscopy registered in the Danish Anesthesia Database. Anesthesiology 2009; 110: 266274. doi: 10.1097/ALN.0b013e318194cac8

7. Hagberg CA, Vogt-Harenkamp C, Kamal J. A retrospective analysis of airway management in obese patients at a teaching institution. J Clin Anesth 2009; 21: 348-351. doi: 10.1016/ j.jclinane.2008.09.009

8. Sheff SR, May MC, Carlisle SE, Kallies KJ, Mathiason MA, Kothari SN. Predictors of a difficult intubation in the bariatric patient: does preoperative body mass index matter? Surg Obes Relat Dis 2013; 9: 344-349. doi: 10.1016/j.soard.2012.02.004

9. Holmberg TJ, Bowman SM, Warner KJ, Vavilala MS, Bulger EM, Copass MK, et al. The association between obesity and difficult prehospital tracheal intubation. Anesth Analg 2011; 112: 1132-1138. doi: 10.1213/ANE.0b013e31820effcc

10. De Jong A, Molinari N, Pouzeratte Y, Verzilli D, Chanques G, Jung B, et al. Dif?cult intubation in obese patients: incidence, risk factors, and complications in the operating theatre and in intensive care units. Br J Anaesth 2015; 114: 297-306. doi: 10.1093/bja/aeu373 
11. Ezri T, Gewürtz G, Sessler DI, Medalion B, Szmuk P, Hagberg C, et al. Prediction of difficult laryngoscopy in obese patients by ultrasound quantification of anterior neck soft tissue. Anaesthesia 2003; 58: 1111-1114. doi: 10.1046/j.1365-2044.2003.03412.x

12. Ezri T, Medalion B, Weisenberg M, Szmuk P, Warters RD, Charuzi I. Increased body mass index per se is not a predictor of difficult laryngoscopy. Can J Anaesth 2003; 50: 179-183. doi: 10.1007/BF03017853

13. Kim WH, Ahn HJ, Lee CJ, Shin BS, Ko JS, Choi SJ, et al. Neck circumference to thyromental distance ratio: a new predictor of difficult intubation in obese patients. Br J Anaesth 2011; 106: 743-748. doi: 10.1093/bja/aer024

14. Gonzalez H, Minville V, Delanoue K, Mazerolles M, Concina D, Fourcade O. The importance of increased neck circumference to intubation difficulties in obese patients. Anesth Analg 2008; 106: 1132-1136. doi: 10.1213/ane.0b013e3181679659

15. Juvin P, Lavaut E, Dupont H, Lefevre P, Demetriou M, Dumoulin JL, et al. Difficult tracheal intubation is more common in obese than in lean patients. Anesth Analg 2003; 97: 595-600. doi: 10.1213/01.ANE.0000072547.75928.B0

16. Voyagis GS, Kyriakis KP, Dimitriou V, Vrettou I. Value of oropharyngeal Mallampati classification in predicting difficult laryngoscopy among obese patients. Eur J Anaesthesiol 1998; 15: 330-334. doi:10.1046/j.1365-2346.1998.00301.x

17. Neligan PJ, Porter S, Max B, Malhotra G, Greenblatt EP, Ochroch EA. Obstructive sleep apnea is not a risk factor for difficult intubation in morbidly obese patients. Anesth Analg 2009; 109: 1182-1186. doi:0 10.1213/ane.0b013e3181b12a0c

18. Ezri T, Warters RD, Szmuk P, Saad-Eddin H, Geva D, Katz J, et al. The incidence of class "zero" airway and the impact of
Mallampati score, age, sex, and body mass index on prediction of laryngoscopy grade. Anesth Analg 2001; 93: 1073-1075. doi: 10.1097/00000539-200110000-00055

19. Marrel J, Blanc C, Frascarolo P, Magnusson L. Videolaryngoscopy improves intubation condition in morbidly obese patients. Eur J Anaesthesiol 2007; 24: 1045-1049. doi: 10.1017/ S0265021507000889

20. Bathory I, Granges JC, Frascarolo P, Magnusson L. Evaluation of the Video Intubation Unit in morbid obese patients. Acta Anaesthesiol Scand 2010; 54: 55-58. doi: 10.1111/j.13996576.2009.02119.x

21. Aziz MF, Brambrink AM, Healy DW, Willett AW, Shanks A, Tremper T, et al. Success of Intubation Rescue Techniques after Failed Direct Laryngoscopy in Adults: A Retrospective Comparative Analysis from the Multicenter Perioperative Outcomes Group. Anesthesiology 2016; 125: 656-666. doi: 10.1097/ALN.0000000000001267

22. Asai T. Avoiding repeated attempts at tracheal intubation. Can videolaryngoscopes be the answer? Anesthesiology 2016; 125: 615-617. doi: 10.1097/ALN.0000000000001268

23. Fox WTA, Harris S, Kennedy NJ. Prevalence of difficult intubation in a bariatric population, using the beach chair position. Anaesthesia 2008; 63: 1339-1342. doi: 10.1111/j.13652044.2008.05639.x

24. Rao SL, Kunselman AR, Schuler HG, DesHarnais S. Laryngoscopy and tracheal intubation in the head-elevated position in obese patients: a randomized, controlled, equivalence trial. Anesth Analg 2008; 107: 1912-1918. doi: 10.1213/ane.0b013e $31818556 \mathrm{ed}$ 